This is the pre-peer reviewed version of the following article:

Šišoláková I., Hovancová J., Orinaková R., Orinak A., Rueda Garcia D., Shylenko O., Radonák J.. Comparison of Insulin Determination on NiNPs/chitosan- MWCNTs and NiONPs/chitosan-MWCNTs Modified Pencil Graphite Electrode. Electroanalysis, (2019). 31. : 103 - .

10.1002/elan.201800483,

which has been published in final form at https://dx.doi.org/10.1002/elan.201800483. This article may be used for non-commercial purposes in accordance with Wiley Terms and Conditions for Use of Self-Archived Versions. 


\title{
Comparison of insulin determination on NiNPs/chitosan- MWCNTs and NiONPs/chitosan-MWCNTs modified pencil graphite electrode
}

\author{
Ivana Šišolákováa , Jana Hovancováa, Renáta Oriňakováa ${ }^{*}$, Andrej Oriňak ${ }^{a}$, Daniel Rueda Garcia ${ }^{b}$, Oleg Shylenko ${ }^{c}$ \\ Jozef Radoňák ${ }^{d}$ \\ Department of Physical Chemistry, University of P.J. Šafárik in Košice, Moyzesova 11, 04001 Košice, Slovakia \\ b Institut de Ciència de Materials de Barcelona, Campus UAB, E- 08193 Bellaterra, Barcelona, Spain \\ c Institute of Physics, University of P.J. Šafárik in Košice, Park Angelinum 9, 04001 Košice, Slovakia \\ Faculty of medicine, University of P.J. Šafárik in Košice, Trieda SNP 1, 04001 Košice, Slovakia \\ * Corresponding author: renata.orinakova@upjs.sk mailto:mstreckova@imr.saske.sk \\ Phone number: +421-55-2342324 \\ Fax: $\quad+421-55-6222124$
}

\begin{abstract}
The rising amount of patients suffering for diabetes mellitus increases the requirements for effective insulin sensors. Carbon materials are a suitable choice for the development of insulin sensors due to their electrochemical characteristics. Pencil graphite electrodes (PGE) represent the trade-off between price and excellent conductive properties. The modification of PGE by NiO and Ni nanoparticles fixed by chitosan results in surface area enlargement and improved electrocatalytic properties. This paper is focused on the comparison of different properties of $\mathrm{Ni}$ and $\mathrm{NiO}$ nanoparticles and their effect on redox reaction mechanism of insulin and detection characteristics. The electrode modified by Ni nanoparticles displays linear range of $1 \mu \mathrm{M}-5 \mu \mathrm{M}\left(R^{2} 0.80\right)$, limit of detection (LOD) of $4.34 \mu \mathrm{M}$ and sensitivity of $0.12 \mu \mathrm{A} / \mu \mathrm{M}$. On the other hand, the electrode modified by $\mathrm{NiO}$ nanoparticles displays enhanced electrochemical characteristics such as linear range of $0.05 \mu \mathrm{M}-5 \mu \mathrm{M}\left(R^{2} 0.99\right)$, limit of detection of $260 \mathrm{nM}$ and sensitivity of $0.64 \mu \mathrm{A} / \mu \mathrm{M}$. These properties make the $\mathrm{NiO}$ nanoparticles modified PGE the appropriate candidate for insulin determination.
\end{abstract}

Keywords: insulin, electrochemical determination, pencil graphite electrodes, nickel nanoparticles, nickel oxide nanoparticles 


\section{Introduction}

Insulin is a polypeptide hormone that consists of 51 amino acids divided into A and $\mathrm{B}$ chain linked by two disulphide bridges. A and B chains are composed of 21 and 30 residues, respectively. It is produced by $\beta$ cells located in the pancreatic arrays called islets of Langerhans [1] and the normal fasting insulin level in blood reaches maximum value of $25 \mathrm{mIU} / 1(0.86 \mu \mathrm{M})$ [2]. Insulin ensures transport of blood glucose to the cells and controls glucose level in blood [3, 4] The dysfunction of insulin hormone production causes the very common disease in the world called diabetes mellitus [5,6].

Therefore it is necessary to focus on development of cheap, fast and exact insulin sensor [7]. There is a variety of analytical methods used for insulin determination which can be classified into two main groups, immune and non-immune [8]. The most frequently used immune methods are radioimmunoassay (RIA) [9], enzyme immunoassay (EIA) [10] and luminescent immunoassay (LIA) [11]. The main disadvantages of these methods are long-lasting analysis and low sensitivity [12], which can be increased by derivatization of insulin with isotopes and florigenic labels [13]. Non-immune methods such as high performance liquid chromatography (HPLC) $[14,15]$ and capillary electrophoresis (CE) [16] require expensive instrumentation, long lasting analysis and complex pre-treatment steps [17]. Among the various methods that have been used for insulin detection, electrochemical assays are still considered the best methods for insulin determination, because they could overcome the shortcomings of the other methods mentioned above [18]. The main advantages of electrochemical methods are low detection limit, wide linear range, low cost instruments and high sensitivity [19].

The most commonly used electrode material for insulin determination is carbon [20]. Different types of carbon electrodes such as glassy carbon electrodes [21], screen printed carbon electrodes [22], pencil graphite electrodes [23] and carbon paste electrodes [24] have been used for insulin determination. In general, PGE consists of graphite and clay. PGEs have several advantages in comparison to other carbon-based electrodes, including, very low cost, simple modification, widespread availability [25] and well defined surface area [11]. Despite carbon being considered the most suitable material for insulin determination, the bare unmodified carbon electrodes have some drawbacks which can be eliminated by modification with different nanostructures [26]. Therefore, various materials for electrode modification such as multiwalled carbon nanotubes (MWCNTs), metal nanoparticles, metal oxide nanoparticles or combination of these materials have been studied. MWCNTs are frequently used in electrochemical determination of insulin for electrode modifications due to their mechanical stability and fast electron transport. MWCNTs are also known as an excellent option to enlarge electrode surface area, create more active sites for insulin oxidation, increase voltammetric current and decrease overpotential [27]. It is also well known that large surface area of MWCNTs combined with metal nanoparticles or metal oxide nanoparticles can improve the performance of the final electrode material [28, 29].

For this reason, different combinations of MWCNTs and different types of metal nanoparticles like silver nanoparticles [30], silica nanoparticles [8, 12, 31], ruthenium oxide nanoparticles [32], nickel nanoparticles (NiNPs) [33] and nickel oxide nanoparticles (NiONPs) have been studied [29]. Nickel based nanomaterials have 
become an intensively studied material not only because of their low cost in comparison with $\mathrm{Ag}$, Co, $\mathrm{Pd}$ or Pt nanoparticles but also because they possess some desirable characteristics such as high electrical conductivity and good electrocatalytic activity [34]. It is known that the chemical and physical properties of the NiONPs and NiNPs are quite different from bulk Ni and NiO crystals. Particularly, large surface area and excellent magnetic properties are the greatest advantages of synthetized nanoparticles [27]. In addition, it has been revealed that many nickel-based materials exhibit excellent electrocatalytic activity towards the oxidation of a wide range of small compounds, like insulin or glucose in alkaline media [35]. The utilisation of polymer membrane such as Nafion, chitosan, polyethylene glycol prevents the fast occupation of active sites at higher insulin concentration and occupation of active sites by chloride ions in phosphate buffered saline (under physiological condition) [13]. These polymers are also used because of their ability to fix nanoparticles on the electrode surface during electrochemical measurements [36].

In this paper, we have studied the electrocatalytic activity of the NiONPs/chitosan-MWCNTs/PGE and NiNPs/chitosan-MWCNTs/PGE prepared by pulsed electrochemical deposition of NiONPs or NiNPs on chitosan-MWCNTs modified PGE surface towards insulin oxidation. The electrochemical properties of modified PGEs were studied by cyclic voltammetry, chronoamperometry and electrochemical impedance spectroscopy (EIS) in phosphate buffered saline solution simulating human body conditions. The surface of prepared electrodes was characterized by transmission electron microscopy (TEM), scanning transmission electron microscopy (STEM) and EDX analysis. Both of prepared electrodes showed good electrocatalytic activity towards insulin oxidation. The NiONPs/chitosan-MWCNTs/PGE had better electrochemical characteristics and wider linear range when compared to electrochemical characteristics and linear range of NiNPs/chitosanMWCNTs/PGE, what makes it a suitable candidate for electrochemical insulin sensor.

\section{Experimental}

\subsection{Chemicals and Materials}

The multiwalled carbon nanotubes (diameter $3 \mathrm{~nm}-10 \mathrm{~nm}$ and $1 \mu \mathrm{m}-10 \mu \mathrm{m}$ length) were purchased from BOC SCIENCES Creative Dynamics Inc. (USA, NY). Insulin Human Recombinant (5807.6 g/mol) was purchased from MP Biomedicals (France, Illkrich). Phosphate buffered saline (PBS D8662, sterile filtered), sodium hydroxide $(\mathrm{NaOH}, 99 \%)$, nickel nitrate hexahydrate $\left(\mathrm{Ni}(\mathrm{NO})_{3} .6 \mathrm{H}_{2} \mathrm{O}, 99.9 \%\right)$, potassium hexacyanoferrate trihydrate $\left(\mathrm{K}_{4}\left[\mathrm{Fe}(\mathrm{CN})_{6}\right] .3 \mathrm{H}_{2} \mathrm{O}, 99.95 \%\right)$, nitric acid $\left(\mathrm{HNO}_{3}, 65 \%\right)$ and sulfuric acid $\left(\mathrm{H}_{2} \mathrm{SO}_{4}\right.$, 96\%) were purchased from Sigma Aldrich (USA, Missouri). Insulin solutions were freshly prepared by dissolving powdered insulin in $0.1 \mathrm{M} \mathrm{NaOH}$ in PBS before every electrochemical measurement. All measurements were performed at room temperature and atmospheric pressure.

\section{$2.2 \quad$ Instruments}


All electrochemical experiments were performed by using AUTOLAB type PGSTAT302N (Metrohm, Switzerland) with a three-electrode setup using the bare or modified PGE (Herlitz, Germany) as the working electrode, $\mathrm{Ag} / \mathrm{AgCl}$ (saturated $\mathrm{KCl}$ ) as the reference electrode and platinum electrode as the counter electrode. The structure and surface morphology of the electrodes were characterized by SEM (Jeol JSM 7000 N, Japan) with EDX analysis, TEM and STEM.

\subsection{Preparation of the modified electrode}

The surface of carbon PGE was polished with soft sandpaper (400) and ultrasonically cleaned in ethanol for 1 h. $0.5 \mathrm{~g}$ of MWCNTs were suspended in $80 \mathrm{ml}$ of solution containing nitric and sulfuric acid (3:1). $10 \mathrm{ml}$ of ethanol was slowly dropped into the stirred suspension. The mixture was then refluxed for $120 \mathrm{~min}$. The MWCNTs were filtered off and dried at $70^{\circ} \mathrm{C}$ for $5 \mathrm{~h}$ [37]. $10 \mathrm{mg}$ of activated MWCNTs were dispersed in $5 \mu 1$ of chitosan and $5 \mathrm{ml}$ of deionized water and ultrasonicated for $1 \mathrm{~h}$ to get homogenous suspension. $10 \mu \mathrm{l}$ of the suspension were dropped onto the polished surface of carbon pencil electrode and dried at room temperature for $60 \mathrm{~min}$.

The deposition conditions for NiONPs were chosen according to B. Rafiee et al. [29]. The deposition of NiONPs was performed from a $40 \mathrm{mM} \mathrm{Ni}\left(\mathrm{NO}_{3}\right)_{2} \cdot 6 \mathrm{H}_{2} \mathrm{O}$ solution with $\mathrm{pH}=2$. The $\mathrm{pH}$ of the solution was adjusted using sulfuric acid. The pulsed electrodeposition of NiONPs on the PGE was carried out using an optimized double-pulse sequence of potentials: $E_{1}=0.4 \mathrm{~V}$ (vs. $\mathrm{Ag} / \mathrm{AgCl} / \mathrm{KCl}$ ) for $t_{1}=0.3 \mathrm{~s}$ and $E_{2}=0.0 \mathrm{~V}$ (vs. $\mathrm{Ag} / \mathrm{AgCl} / \mathrm{KCl}$ ) for $t_{2}=2.5 \mathrm{~s}$. Electrochemical deposition of NiNPs was performed by applying constant potential $E_{l}$ for time $t_{l}$. Prepared electrodes were activated in $0.1 \mathrm{M} \mathrm{NaOH}$ solution using cyclic voltammetry method with potential cycling between $100 \mathrm{mV}$ and $700 \mathrm{mV}$ for 10 cycles at the scan rate of $100 \mathrm{mV} / \mathrm{s}$. This step was applied for maximum activation of electrode surface towards insulin oxidation [29].

\subsection{Electrochemical determination of active surface of bare PGE and modified electrodes}

The active surface area of bare PGE, chitosan-MWCNTs/PGE, NiNPs/chitosan-MWCNTs/PGE and NiONPs/chitosan-MWCNTs/PGE was determined using double potential step chronoamperometry method in a solution consisting of $1 \mathrm{mM} \mathrm{K}_{4}\left[\mathrm{Fe}(\mathrm{CN})_{6}\right]$ and $0.1 \mathrm{M} \mathrm{NaCl}$. The applied potential steps were $E_{l}=$ open circuit potential for each electrode (176 mV for bare PGE, $132 \mathrm{mV}$ for chitosan-MWCNTs/PGE, -54 mV for NiNPs/chitosan-MWCNTs/PGE and $-42 \mathrm{mV}$ for NiONPs/chitosan-MWCNTs/PGE) and $E_{2}=700 \mathrm{mV}$.

\section{$3 \quad$ Results and discussion}

3.1 Morphological characterization of NiNPs/chitosan-MWCNTs/PGE and NiONPs/chitosanMWCNTs/PGE surface 
The surfaces of unmodified chitosan-MWCNTs/PGE and chitosan-MWCNTs/PGE modified with NiNPs and NiONPs were examined by TEM and STEM at different magnifications to get sufficient information about appearance, surface morphology and distribution of metallic nanoparticles. Moreover, the mapping EDX analysis was used to characterise the surface chemical composition of both modified electrodes. Figure 1 shows the TEM image of chitosan-MWCNTs/PGE without NiNPs or NiONPs. The MWCNTs with diameter of $10 \mathrm{~nm}-20 \mathrm{~nm}$ and several micrometres in length can be seen in Figure 1. The surface of unmodified MWCNTs covered by a chitosan layer is smooth.

Figure 2 shows the TEM (A, D) and STEM (B, C, E, F) images of modified electrodes together with EDX elemental maps of carbon and nickel and X-ray spectrum $(\mathrm{G}, \mathrm{H})$ expressing the elemental composition of the surface of NiNPs/chitosan-MWCNTs/PGE (A, B, C, G) and NiONPs/chitosan-MWCNTs/PGE (D, E, F, H). Before TEM images were obtained all samples were placed on copper grids which caused unequal distribution of MWCNTs on the grid surface (Fig. 2 A, D). TEM images (Fig. 2 A, D) show that the surface of MWCNTs became coarser after loading with NiNPs and NiONPs in comparison with chitosan-MWCNTs surface (Fig. 1). Also, detailed STEM images of NiNPs/chitosan-MWCNTs/PGE (Fig. 2 B, C) and NiONPs/chitosanMWCNTs/PGE (Fig. 2 E, F) show that NiNPs and NiONP were deposited on the surface of chitosan-MWCNTs. NiNPs and NiONPs are mainly distributed on the ruggedness of the chitosan-MWCNTs surface. The size and shape of deposited NiNPs and NiONPs is very similar. The spherical NiNPs and NiONPs are $2 \mathrm{~nm}-5 \mathrm{~nm}$ in diameter without generation of aggregates. The chemical composition of the NiNPs/chitosan-MWCNTs/PGE (Fig. 2 G) and NiONPs/chitosan-MWCNTs/PGE (Fig. 2 H) surface was determined by EDX analyses. The EDX spectrum confirmed the nickel nanoparticles deposition and the presence of magnesium ( $\mathrm{Mg})$, calcium (Ca), silicon ( $\mathrm{Si}$ ), phosphorus (P) and iron (Fe) was caused by pencil substrate composition. Figure 2 shows successful electrodeposition of NiONPs and NiNPs at chosen potentials with similar size $2 \mathrm{~nm}-5 \mathrm{~nm}$. Also the amount of deposited Ni on the electrode surface was favourable, $16.64 \%$ and $20.92 \%$ for NiNPs/chitosan-MWCNTs/PGE and NiONPs/chitosan-MWCNTs/PGE, respectively.

\subsection{Electrocatalytic oxidation of insulin}

Electrochemical behaviour of various PGE surfaces towards insulin oxidation in $0.1 \mathrm{M} \mathrm{NaOH}$ and PBS was studied using cycling voltammetry method $(\mathrm{CV})$ in a potential window ranging from $-1 \mathrm{~V}$ to $2 \mathrm{~V}$ with a scan rate of $100 \mathrm{mV} / \mathrm{s}$. Fig. 3 shows cyclic voltammograms recorded in $0.1 \mathrm{M} \mathrm{NaOH}$ and PBS for bare PGE in absence of insulin (a) and in the presence of $2 \mu \mathrm{M}$ insulin for bare PGE (b) chitosan-MWCNTs/PGE (c), NiONPs/chitosan-MWCNTs/PGE (d), and NiNPs/chitosan-MWCNTs/PGE (e).

As shown in these voltammograms, current response towards insulin oxidation on bare PGE (Fig. 3 b) is significantly lower in comparison to current response towards insulin oxidation on chitosan-MWCNTs/PGE (Fig. 3 c), NiONPs/chitosan-MWCNTs/PGE (Fig. 3 d) and NiNPs/chitosan-MWCNTs/PGE (Fig. 3 e). There is also no apparent peak found for the bare PGE (Fig. 3 b), confirming that the bare PGE has no significant electrocatalytic 
activity towards insulin in aqueous alkaline solutions. Two oxidation peaks were observed in cyclic voltammograms for NiONPs/chitosan-MWCNTs/PGE (d), and NiNPs/chitosan-MWCNTs/PGE (e) because of the two-step oxidation process on the modified electrode. Moreover, two reduction peaks were detected for both the electrodes, but the difference in the values of oxidation peaks potential indicates the different mechanism of oxidation process.

Firstly, the current response of NiONPs/chitosan-MWCNTs/PGE is significantly lower in comparison with this of the NiNPs/chitosan-MWCNTs/PGE. The mechanism of insulin oxidation on NiONPs/chitosanMWCNTs/PGE can be shown as follows:

$$
\begin{aligned}
& \mathrm{NiO}+\mathrm{OH}^{-} \rightarrow \mathrm{NiO}(\mathrm{OH})+\mathrm{e}^{-} \\
& \mathrm{NiO}(\mathrm{OH})+\text { insulin } \rightarrow \mathrm{NiO}+\text { product }
\end{aligned}
$$

The oxidative current peaks at $1.2 \mathrm{~V}$ and $1.45 \mathrm{~V}$ (Fig. $3 \mathrm{~d}$ ) correspond to the reactions expressed by equations 1 and 2, respectively.

Secondly, NiNPs/chitosan-MWCNTs/PGE displays enhanced current response and oxidation potentials shifted to lower values $(0.6 \mathrm{~V}$ and $1.1 \mathrm{~V})$, as a consequence of different electroactive species. The shifted oxidative current peaks at $0.6 \mathrm{~V}$ and $1.1 \mathrm{~V}$ (Fig. 3 e) correspond to the reactions expressed by equations $3-5$ :

$$
\begin{aligned}
& \mathrm{Ni}+2 \mathrm{OH}^{-} \rightarrow \mathrm{Ni}(\mathrm{OH})_{2}+2 \mathrm{e}^{-} \\
& \mathrm{Ni}(\mathrm{OH})_{2}+\mathrm{OH}^{-} \rightarrow \mathrm{NiO}(\mathrm{OH})+\mathrm{H}_{2} \mathrm{O}+\mathrm{e}^{-} \\
& \mathrm{NiO}(\mathrm{OH})+\text { insulin } \rightarrow \mathrm{Ni}(\mathrm{OH})_{2}+\text { product }
\end{aligned}
$$

The $\mathrm{NiO}(\mathrm{OH})$ species present during the electrochemical oxidation of insulin on both electrode surfaces, catalyse the oxidation of insulin and enhance current response when compared to bare electrode [29].The distance between oxidative and redox peaks in both cases indicates irreversible electrochemical oxidation of insulin.

These measurements confirm better electrocatalytic activity of NiONPs/chitosan-MWCNTs/PGE and NiNPs/chitosan-MWCNTs/PGE towards insulin oxidation compared to bare PGE or chitosan-MWCNTs/PGE. Through the obtained voltammograms was the mechanism of insulin oxidation on NiONPs/chitosanMWCNTs/PGE and NiNPs/chitosan-MWCNTs/PGE described (Eguation 1 - 5).

\subsection{Effect of scan rate and active surface area of working electrode}

The effect of scan rate on the electrochemical behaviour was studied in $0.1 \mathrm{M} \mathrm{NaOH}$ and PBS for NiONPs/chitosan-MWCNTs/PGE (Fig. 4 A, C) and NiNPs/chitosan-MWCNTs/PGE (Fig. 4 B, D) in the presence of $2 \mu \mathrm{M}$ insulin. In both cases the anodic peak current exhibited linear increase with the rising scan rate, with correlation coefficient $R^{2} 0.99$ and 0.95 for NiONPs/chitosan-MWCNTs/PGE and NiNPs/chitosanMWCNTs/PGE, respectively. The linear regression equations are:

$$
\begin{aligned}
& I_{p a}(\text { NiONPs } / \text { chitosan - MWCNTs } / \text { PGE })=0.00303 v(\mathrm{mV} / \mathrm{s})+0.37 \\
& I_{p a}(\text { NiNPs } / \text { chitosan - MWCNTs } / \text { PGE })=2.0292 .10^{-5} v(\mathrm{mV} / \mathrm{s})+1.84 .10^{-4}
\end{aligned}
$$


According these results, the electron transfer between the redox sites of both electrode surfaces is a typical surface-controlled process. The rate determining step is the charge transfer on electrode surface.

Enlargement of the electrode surface area due to the deposition of nanoparticles increases active surface area and number of active sites. The active surface area of electrode was measured by chronoamperometry and calculated according to the Cottrell equation:

$$
i=\frac{n F A D^{1 / 2} C}{\pi^{1 / 2} t^{1 / 2}}
$$

where $i$ is measured current, $n$ is number of exchanged electrons, $F$ is a Faraday constant, $A$ is a surface area of working electrode, $C$ a concentration of $\mathrm{K}_{4}\left[\mathrm{Fe}(\mathrm{CN})_{6}\right]$ and $t$ is the time in seconds. Chronoamperograms for bare PGE (a) chitosan-MWCNTs/PGE (b), NiONPs/chitosan-MWCNTs/PGE (c), and NiNPs/chitosanMWCNTs/PGE (d) were obtained in $1 \mathrm{mM} \mathrm{K}_{4}\left[\mathrm{Fe}(\mathrm{CN})_{6}\right]$ and $0.1 \mathrm{M} \mathrm{NaCl}$ and the plots of oxidation peak current versus $t^{1 / 2}$ were fitted by linear function (Fig. 5 inset). The active surface areas were $0.33 \mathrm{~mm}^{2}$ for bare electrode, $1.33 \mathrm{~mm}^{2}$ for chitosan-MWCNTs/PGE, $1.34 \mathrm{~mm}^{2}$ for NiONPs/chitosan-MWCNTs/PGE and $1.39 \mathrm{~mm}^{2}$ for NiNPs/chitosan-MWCNTs/PGE. After modification the active surface area increased more than 4 times, what represents rapid enlargement of active surface area of modified electrodes. The value of the diffusion coefficient of insulin was calculated on the basis of Cottrell equation and measurement was realized for $2 \mu \mathrm{M}$ insulin and 0.1 $\mathrm{M} \mathrm{NaOH}$ in PBS. The surface area was $0.33 \mathrm{~mm}^{2}$ and $n=1$. The calculated diffusion coefficient was $0.08 .10^{-6}$ $\mathrm{cm}^{2} / \mathrm{s}$.

\subsection{Electrochemical impedance spectroscopy}

EIS is important analytical tool which was used to study the catalytic procedure of insulin electrooxidation on different electrode surfaces. Fig. 6 shows Nyquist plots recorded at $1.45 \mathrm{~V}$ in frequency range of $0.1 \mathrm{~Hz}$ to $100000 \mathrm{~Hz}$ in presence of $2 \mu \mathrm{M}$ insulin and $0.1 \mathrm{M} \mathrm{NaOH}$ in PBS. The most suitable circuit that describes electrochemical system is depicted in Inset in Figure 6. $R_{S}$ is the solution resistance, $C_{d l}$ is double-layer capacitance and $R_{c t}$ is charge transfer reaction resistance. The increase in semicircle diameter in the impedance spectrum represents the decrease in electron-transfer resistance indicating the enhanced electrochemical redox reaction on the surface of the electrode.

As shown in Fig. 6, the largest semicircle was observed for bare PGE (a), which correspond to the surface with the lowest conductivity. Higher conductivity was achieved by modification of PGE surface with MWCNTs (c), while the conductivity decreased after addition of chitosan (b). Opposite trend was observed in case of comparison of chitosan-MWCNTs/NiNPs (e) with MWCNTs/NiNPs (d), where chitosan application caused the decrease in $R_{c t}$ and improves the charge transfer efficiency at electrolyte-electrode interface. This effect is associated with the stabilization of NiNPs on MWCNTs PGE surface with chitosan resulting in increased conductivity. The same phenomenon was observed for NiONPs/MWCNTs/PGE (f) and NiONPs/chitosanMWCNTs/PGE (g). In conclusion, the electrode modified by NiONPs and NiNPs fixed with chitosan enhanced conduction pathway with higher electron transfer. Among them, the NiONPs exhibited higher conductivity and 
electrocatalytic activity. To evaluate the effect of insulin concentration on EIS measurement, the Nyquist diagrams at different insulin concentrations $(1 \mu \mathrm{M}-5 \mu \mathrm{M})$ in $0.1 \mathrm{M} \mathrm{NaOH}$ in PBS on NiNPs/chitosanMWCNTs/PGE and NiONPs/chitosan-MWCNTs/PGE surface were obtained.

The decrease in semicircles diameter with rising insulin concentration can be seen from the Nyquist plots of both NiNPs/Chitosan-MWCNTs/PGE (Fig. 7 A) and NiONPs/Chitosan-MWCNTs/PGE (Fig. 7 B) recorded at $1.45 \mathrm{~V}$. Insets in Fig. $7 \mathrm{~A}$ and B-show the insulin concentration dependence on $R_{c t}$ fitted by linear function. The value of $R_{c t}$ decreased with rising insulin concentration. Correlation coefficients were 0.89 and 0.95 for NiNPs/chitosan-MWCNTs/PGE and NiONPs/chitosan-MWCNTs/PGE surfaces, respectively. It follows from these results, that the electrochemical response of NiONPs/chitosan-MWCNTs/PGE is more linear in comparison with NiNPs/chitosan-MWCNTs/PGE.

\subsection{Effect of different insulin concentration}

On the basis of the voltammetric results described above, the NiONPs/chitosan-MWCNTs/PGE and NiNPs/chitosan-MWCNTs/PGE appears to be a suitable sensor for the sensitive determination of insulin. Fig. 8 shows the effect of the various concentrations of insulin $(0.05 \mu \mathrm{M}-5 \mu \mathrm{M})$ on anodic peak current $(1.45 \mathrm{~V})$ at the NiONPs/chitosan-MWCNTs/PGE in $0.1 \mathrm{M} \mathrm{NaOH}$ and PBS solution. The anodic peak current increased linearly by rising of the insulin concentration, what was confirmed by determination of the correlation coefficient $\left(R^{2}\right.$ 0.99). The plot of current response versus insulin concentration is linear over the wide concentration range of $0.05 \mu \mathrm{M}-5 \mu \mathrm{M}$ (Fig. 8, inset). The sensitivity of modified electrode emerged from the calibration plot with the correlation coefficient of 0.99 was $0.64 \mu \mathrm{A} / \mu \mathrm{M}$. The limit of detection, evaluated at a signal to noise ratio of 3:2, was found to be $260 \mathrm{nM}$. The linear calibration range, sensitivity and detection limit for insulin determination at NiONPs/chitosan-MWCNTs/PGE are comparable with those obtained by common modified electrodes (Tab. 1).

Also correlation coefficient was similar like correlation coefficients obtained by common modified electrode mentioned in Tab. 1 [25, 38, 39].

Oxidation of insulin $(0.05 \mu \mathrm{M}-5 \mu \mathrm{M})$ in $0.1 \mathrm{M} \mathrm{NaOH}$ and PBS on the NiNPs/chitosan-MWCNTs/PGE surface was also studied. As shown in cyclic voltammograms of insulin on NiNPs/chitosan-MWCNTs/PGE surface (Fig. 9), the anodic peak current (1.1 V) was slightly shifted to lower values, compared to anodic peak current of insulin oxidation on NiONPs/chitosan-MWCNTs/PGE (1.45 V). The plot of current response versus insulin concentration was linear only over the concentration range of $1 \mu \mathrm{M}-5 \mu \mathrm{M}$ (Fig. 9, inset), which was lower concentration range in comparison with linear concentration range obtained for NiONPs/chitosanMWCNTs/PGE $(0.05 \mu \mathrm{M}-5 \mu \mathrm{M})$ (Fig. 8). Moreover, the sensitivity $(0.12 \mu \mathrm{A} / \mu \mathrm{M})$ was lower, the correlation coefficient was insufficient $\left(R^{2} 0.78\right)$ and linearity of calibration plot deteriorated. The limit of detection, evaluated at a signal to noise ratio of 3:2, was found to be $4.34 \mu \mathrm{M}$ (Fig. 9, inset). Further this characteristic of NiNPs/chitosan-MWCNTs/PGE surface was worse as compared to characteristic obtained for 
NiONPs/chitosanMWCNTs /PGE. According to these results, it can be concluded, that NiONPs particles possess better electrocatalytic properties and are most suitable material for insulin determination.

Tab. 1 provides the comparison of limits of detection, sensitivity and linear dynamic range towards insulin oxidation for different modified carbon electrodes. For all mentioned electrodes the amperometry method of insulin determination was used. As can be seen in Tab. 1 the prepared NiONPs/chitosan-MWCNTs/PGE possessed the widest dynamic range in comparison with other mentioned electrodes $(0.05 \mu \mathrm{M}-5 \mu \mathrm{M})$. NiONPs/chitosan-MWCNTs modification of PGE also resulted in an excellent limit of detection (260 nm), which was significantly lower than in the case of glassy carbon electrode modified with MWCNTs and dihydropyran (1 $\mu \mathrm{M}$ ) [38] and carbon microelectrode modified with combination of RuO and RuCN (500 $\mathrm{nM}$ ) [32]. Limit of detection of prepared NiONPs/chitosan-MWCNTs/PGE was also comparable with other mentioned electrodes, such as MWCNTs/dimetylformamide/carbon electrode (250 nM) [39] and NiNP/CNTs-modified carbon fibermicroelectrode (270 nM) [35]. Prepared NiONPs/chitosan-MWCNTs/PGE had widest linear dynamic range $(0.05 \mu \mathrm{M}-50 \mu \mathrm{M})$ than mentioned electrode. NiONPs/chitosan-MWCNTs/PGE had also good sensitivity (0.64 $\mu \mathrm{A} / \mu \mathrm{M})$ in comparison with other mentioned electrodes mentioned in Tab. 1.

\subsection{Stability of NiONPs/Chitosan-MWCNTs/PGE}

Stability test for NiONPs/chitosan-MWCNTs/PGE and NiNPs/chitosan-MWCNTs/PGE was performed in $1 \mathrm{mM} \mathrm{K}_{4}\left[\mathrm{Fe}(\mathrm{CN})_{6}\right]$ and $0.1 \mathrm{M} \mathrm{NaCl}$ solution in potential range $0.3 \mathrm{~V}-1 \mathrm{~V}$. Obtained cyclic voltemmograms are shown in Figure 10. After 50 cycles, current response decreased by only less than $6 \%$ for NiONPs/chitosanMWCNTs/PGE (Fig. $10 \mathrm{~A}$ ) and by $10.28 \%$ for NiNPs/chitosan-MWCNTs/PGE (Fig. 10 B). The results indicate a better stability for NiONPs in comparison to NiNPs on the chitosan-MWCNTs electrode surface.

Even, insulin with concentration $2 \mu \mathrm{M}$ was determined on five different NiONPs/chitosanMWCNTs/PGEs and a reproducible current response with a great relative standard deviation (RSD) $4.25 \%$ was obtained (Fig. 11).

\section{Conclusion}

A sensitive electrochemical sensor composed of electrodeposited NiONPs nanoparticles on the PGE surface modified with chitosan-MWCNTs was fabricated. Chitosan as a polymer material successfully stabilized the NiONPs on the modified PGE electrode surface. This fact was confirmed by TEM and STEM results and EDX analysis, which indicated that the NiONPs with sizes of about $4 \mathrm{~nm}$ were immobilized on the chitosanMWCNTs surface. The small size of the NiONPs in combination with MWCNTs greatly improved the specific surface area of the PGE and enhanced electrocatalytic activity of PGE towards insulin oxidation. The electroanalytic properties of NiONPs modified electrode were compared with NiNPs/chitosan-MWCNTs/PGE. The NiONPs display much more suitable properties for insulin determination because of wider linear range, improved sensitivity and lower detection limit. All measurements were performed in PBS, represents the 
physiological conditions in human body. All these characteristic determine the NiONPs/chitosan-MWCNTs/PGE as an effective and stable sensor for insulin detection in physiological condition.

\section{$5 \quad$ Acknowledgments}

This work was supported by the Projects APVV-16-0029 of the Slovak Research and Development Agency, VEGA 1/0074/17 of the Slovak Scientific Grant Agency, VVGS-PF-2018-795 and VVGS-PF-2018-794 of Pavol Jozef Šafárik University in Košice.

\section{References}

[1] Y. Wang, J. Li, Anal. Chim. Acta 2009, 650, 49-53.

[2] A. K. Yagati, Y. Choi, J. Park, J. W. Choi, H. S. Jun, S. Cho, Biosens. Bioelectron. 2016, 80, 307-314.

[3] Y. Pu, Z. Zhu, D. Han, H. Liu, J. Liu, J. Liao, K. Zhang, W. Tan, Analyst. 2011, 136, 4138-4140.

[4] Y. Li, L. Tian, L. Liu, M. S. Khan, G. Zhao, D. Fan, W. Cao, Q. Wei, Talanta 2018, 179, 420-425.

[5] N. D. D. Group, Diabetes 1995, 28, 1039.

[6] K. G. M. M. Alberti, P. Z. Zimmet, Diabet. Med. 1998, 15, 539-553.

[7] J. Wang, M. Musameh, Anal. Chim. Acta 2004, 511, 33-36.

[8] A. Salimi, L. Mohamadi, R. Hallaj, S. Soltanian, Electrochem. Commun. 2009, 11, 1116-1119.

[9] M. Frasconi, C. Tortolini, F. Botre, F. Mazzei, F. Botrè, Anal. Chem. 2010, 82, 7335-7342.

[10] W. Bürgi, M. Briner, N. Franken, A. C. Kessler, Clin. Biochem. 1988, 21, 311-314.

[11] T. Tanaka, T. Matsunaga, Anal. Chem. 2000, 72, 3518-3522.

[12] M. Jaafariasl, E. Shams, M. K. Amini, Electrochim. Acta 2011, 56, 4390-4395.

[13] J. Hovancová, I. Šišoláková, R. Oriňaková, A. Oriňak, J. Solid State Electrochem. 2017, 21, 2147-2166.

[14] Y. Luo, K. Huang, H. Xu, Anal. Chim. Acta 2005, 64.

[15] A. Hvass, B. Skelbaek-Pedersen, J. Pharm. Biomed. Anal. 2005, 37, 551-557.

[16] K. Ortner, W. Buchberger, M. Himmelsbach, J. Chromatogr. A 2009, 1216, 2953-2957.

[17] N. F. C. Visser, M. Van Harmelen, H. Lingeman, H. Irth, J. Pharm. Biomed. Anal. 2003, 33, 451-462.

[18] M. Pikulski, W. Gorski, Anal. Chem. 2000, 72, 2696-2702.

[19] B. D. Arya, S. K., Singh, P. S. Malhotra, Electrochemical Techniques in Biosensors 2007.

[20] M. Zhang, C. Mullens, W. Gorski, Anal. Chem. 2005, 77, 6396-6401.

[21] L. Cheng, G. E. Pacey, J. A. Cox, Anal. Chem. 2001, 73, 5607-5610.

[22] J. P. Hart, A. Crew, E. Crouch, K. C. Honeychurch, R. M. Pemberton, Anal. Lett. 2004, 37, 789.

[23] S. Poorahong, C. Thammakhet, P. Thavarungkul, W. Limbut, A. Numnuam, P. Kanatharana, Microchim. Acta 2012, 176, 91.

[24] J. Wang, G. Rivas, X. Cai, M. Chicharro, P. A. M. Farias, E. Palecek, Electroanalysis 1996, 8, 902-906.

[25] M. R. Akanda, M. Sohail, M. A. Aziz, A. N. Kawde, Electroanalysis 2016, 28, 408-424.

[26] B. B. Prasad, R. Madhuri, M. P. Tiwari, P. S. Sharma, Electrochim. Acta 2010, 55, 9146-9156.

[27] E. Martínez-Periñán, M. Revenga-Parra, M. Gennari, F. Pariente, R. Mas-Ballesté, F. Zamora, E. Lorenzo, Sensors Actuators, B Chem. 2016, 222, 331-338.

[28] A. J. S. Ahammad, J.-J. Lee, M. A. Rahman, Sensors 2009, 9, 2289-2319.

[29] B. Rafiee, A. R. Fakhari, Biosens. Bioelectron. 2013, 46, 130-135.

[30] A. Arvinte, A. C. Westermann, A. M. Sesay, V. Virtanen, Sensors Actuators, B Chem. 2010, 150, 756-763.

[31] N. Amini, M. B. Gholivand, M. Shamsipur, J. Electroanal. Chem. 2014, 714-715, 70-75. 
[32] J. Wang, T. Tangkuaram, S. Loyprasert, T. Vazquez-Alvarez, W. Veerasai, P. Kanatharana, P. Thavarungkul, Anal. Chim. Acta 2007, 581, 1-6.

[33] Y. Yu, M. Guo, M. Yuan, W. Liu, J. Hu, Biosens. Bioelectron. 2016, 77, 215-219.

[34] Y. Lin, L. Hu, L. Li, K. Wang, RSC Adv. 2014, 4, 46208-46213.

[35] L. Lu, L. Liang, Y. Xie, K. Tang, Z. Wan, S. Chen, J. Solid State Electrochem. 2018, 22, 825-833.

[36] A. I. Gopalan, K. P. Lee, K. M. Manesh, P. Santhosh, J. H. Kim, J. S. Kang, Talanta, 2007, 71, $1774-1781$.

[37] B. Erdelyi, A. Oriňak, R. Oriňaková, J. Lorinčík, M. Jerigová, D. Velič, M. Mičušík, M. Omastová, R. M. Smith, V. Girman, Appl. Surf. Sci. 2017, 396, 574-581.

[38] R. M. Snider, M. Ciobanu, A. E. Rue, D. E. Cliffel, Anal. Chim. Acta 2008, 609, 44-52.

[39] P. Businova, J. Prasek, J. Chomoucka, J. Drbohlavova, J. Pekarek, R. Hrdy, and J. Hubalek, Procedia Eng. 2012, 47, 12351238 .

Fig. 1. TEM image of chitosan-MWCNTs/PGE

Fig. 2. TEM image of NiNPs/chitosan-MWCNTs/PGE (A), detailed STEM image of NiNPs/Chitosan-MWCNTs/PGE (B, C), EDX maps of $\mathrm{Ni}$ and $\mathrm{C}$, with X-ray spectrum of the NiNPs/Chitosan-MWCNTs/PGE surface (G), TEM image of NiONPs/chitosanMWCNTs/PGE (D), detailed STEM image of NiONPs/Chitosan-MWCNTs/PGE (E, F) and EDX maps of Ni and C with X-ray spectrum of the NiONPs/Chitosan-MWCNTs/PGE surface $(\mathrm{H})$

Fig. 3. Cyclic voltammograms for different electrodes obtained in $0.1 \mathrm{~m} \mathrm{NaOH}$ and PBS in potential range from $-1 \mathrm{~V}$ to $2 \mathrm{~V}$, with the scan rate of $100 \mathrm{mV} / \mathrm{s}$, in the absence of insulin (a) and in the presence of $2 \mu \mathrm{M}$ insulin (b-e): bare PGE (b), chitosanMWCNTs/PGE (c) NiONPs/chitosan-MWCNTs/PGE (d) and NiNPs/chitosan-MWCNTs/PGE (e)

Fig. 4. Cyclic voltammograms for NiONPs/chitosan-MWCNTs/PGE (A) and NiNPs/chitosan-MWCNTs/PGE (B) obtained in 0.1 $\mathrm{m} \mathrm{NaOH}$ and PBS in potential range from $-1 \mathrm{~V}$ to $2 \mathrm{~V}$, with the different scan rates of $25,50,75$ and $100 \mathrm{mV} / \mathrm{s}$. The plot of oxidation peak current versus scan rate for NiONPs/chitosan-MWCNTs/PGE (C) and NiNPs/chitosan-MWCNTs/PGE (D)

Fig. 5. Current transients for bare PGE (a) chitosan-MWCNTs/PGE (b), NiONPs/chitosan-MWCNTs/PGE (c), and NiNPs/chitosan-MWCNTs/PGE (d) obtained in $1 \mathrm{mM} \mathrm{K4}[\mathrm{Fe}(\mathrm{CN}) 6]$ and $0.1 \mathrm{M} \mathrm{NaCl}$. Inset: the plots of oxidation peak current versus $t^{1 / 2}$

Fig. 6. Nyquist diagrams of different modification of PGE surface: unmodified (a), chitosan-MWCNTs/PGE (b), MWCNTs/PGE (c), NiNPs/MWCNTs/PGE (d), NiNPs/chitosan-MWCNTs/PGE (e) NiONPs/MWCNTs/PGE (f) and NiONPs/chitosanMWCNTs/PGE (g) in the presence of $2 \mu \mathrm{M}$ insulin and $0.1 \mathrm{M} \mathrm{NaOH}$ in PBS. Applied potential $1.45 \mathrm{~V}$ and frequency range of 0,1 $\mathrm{Hz}$ to $100000 \mathrm{~Hz}$. Inset: equivalent circuit used for data fitting

Fig. 7. Nyquist diagrams of NiNPs/chitosan-MWCNTs/PGE (A) NiONPs/chitosan-MWCNTs/PGE (B) in the presence of $1 \mu \mathrm{M}$ (a), $2 \mu \mathrm{M}$ (b), $3 \mu \mathrm{M}$ (c), $4 \mu \mathrm{M}$ (d) and $5 \mu \mathrm{M}$ (e) insulin in $0.1 \mathrm{M} \mathrm{NaOH}$ and PBS. Inset: dependency of $\mathrm{R}_{\mathrm{ct}}$ on insulin concentration Fig. 8. Cyclic voltammograms of $0.1 \mathrm{M} \mathrm{NaOH}$ with PBS in the absence (a) and in the presence of $0.05 \mu \mathrm{M}(\mathrm{b}), 0.1 \mu \mathrm{M}(\mathrm{c}), 0.5 \mu \mathrm{M}$ (d), $0.75 \mu \mathrm{M}$ (e), $1 \mu \mathrm{M}$ (f), $2 \mu \mathrm{M}$ (g), $3 \mu \mathrm{M}$ (h), $4 \mu \mathrm{M}$ (i)and $5 \mu \mathrm{M}$ (j) insulin on NiONPs/chitosan-MWCNTs/PGE in potential range $-1 \mathrm{~V}$ to $2 \mathrm{~V}$ with the scan rate of $100 \mathrm{mV} / \mathrm{s}$. Inset: the linear plot of insulin oxidation peak current versus insulin concentration, with correlation coefficient and the straight line equation 
Fig. 9. Cyclic voltammograms of $0.1 \mathrm{M} \mathrm{NaOH}$ with PBS in the absence (a) and in the presence of $0.05 \mu \mathrm{M}(\mathrm{b}), 0.1 \mu \mathrm{M}(\mathrm{c}), 0.5 \mu \mathrm{M}$ (d), $0.75 \mu \mathrm{M}$ (e), $1 \mu \mathrm{M}$ (f), $2 \mu \mathrm{M}$ (g), $3 \mu \mathrm{M}$ (h), $4 \mu \mathrm{M}$ (i) and $5 \mu \mathrm{M}$ (j) insulin on NiNPs/chitosan-MWCNTs/PGE in potential range $-1 \mathrm{~V}$ to $2 \mathrm{~V}$ with the scan rate of $100 \mathrm{mV} / \mathrm{s}$. Inset: the linear plot of insulin oxidation peak current versus insulin concentration, with correlation coefficient and the straight line equation

Fig. 10. Cyclic voltammograms of 1 st (a) and 50th (b) cycle of determination of $1 \mathrm{mM} \mathrm{K}_{4}\left[\mathrm{Fe}(\mathrm{CN})_{6}\right]$ and $0.1 \mathrm{M} \mathrm{NaCl}$ on NiONPs/chitosan-MWCNTs/PGE (A) and NiNPs/chitosan-MWCNTs/PGE (B) in potential range $0.3 \mathrm{~V}$ to $1 \mathrm{~V}$ with the scan rate of $100 \mathrm{mV} / \mathrm{s}$

Fig. 11 Cyclic voltammogram of determination of $2 \mu \mathrm{M}$ insulin in $0.1 \mathrm{M} \mathrm{NaOH}$ and PBS on five different NiONPs/chitosanMWCNTs/PGE

Table 1 Comparision of analytical parameters for insulin determination with several modified electrodes

\begin{tabular}{|c|c|c|c|c|c|}
\hline Electrode & Method & LOD & Sensitivity & $\begin{array}{l}\text { Dynamic } \\
\text { range }\end{array}$ & Reference \\
\hline $\begin{array}{l}\text { NiONPs/Chitosan- } \\
\text { MWCNTs/PGE [a] }\end{array}$ & Amperometry & $260 \mathrm{nM}$ & $0.64 \mu \mathrm{A} / \mu \mathrm{M}$ & $0.05 \mu \mathrm{M}-5 \mu \mathrm{M}$ & This work \\
\hline DHP [b] /MWCNTs/GCE [c] & Amperometry & $1 \mu \mathrm{M}$ & $1.33 \mathrm{nA} / \mu \mathrm{M}$ & $\begin{array}{l}0.8 \mu \mathrm{M}-2.5 \\
\mu \mathrm{M}\end{array}$ & [38] \\
\hline CNTs/GCE & Amperometry & $\begin{array}{l}0.15 \\
\mu \mathrm{M}\end{array}$ & - & $\begin{array}{l}1.5 \mu \mathrm{M}-2.5 \\
\mu \mathrm{M}\end{array}$ & {$[1]$} \\
\hline MWCNTs/DMF [d] /CE [e] & Amperometry & $250 \mathrm{nM}$ & - & $\begin{array}{l}250 \mathrm{nM}-1.6 \\
\mu \mathrm{M}\end{array}$ & [39] \\
\hline $\mathrm{RuO} / \mathrm{RuCN} / \mathrm{CME}[\mathrm{f}]$ & Amperometry & $500 \mathrm{nM}$ & $441 \mu \mathrm{A} / \mu \mathrm{M}$ & - & [32] \\
\hline $\begin{array}{l}\text { MWCNTs- } \\
\text { Nafion/NiONPs/SCPE [g] }\end{array}$ & Amperometry & $6.1 \mathrm{nM}$ & $1.83 \mu \mathrm{A} / \mu \mathrm{M}$ & $\begin{array}{l}20 \mathrm{nM}-260 \\
\mathrm{nM}\end{array}$ & [29] \\
\hline $\begin{array}{l}\text { NiNP/CNTs-modified carbon } \\
\text { fiber } \\
\text { microelectrode }\end{array}$ & Amperometry & $270 \mathrm{nM}$ & $1.11 \mathrm{nA} / \mu \mathrm{M}$ & $2 \mu \mathrm{M}-20 \mu \mathrm{M}$ & {$[35]$} \\
\hline
\end{tabular}

[a] Pencil graphite electrode

[b] Dihydropyran

[c] Glassy carbon electrode

[d] Dimetylformamide [e] Carbon electrode

[f] Carbon microelectrode

[g] Screen printed carbon electrode 